\title{
La Façon de Venise en Castilla
}

\author{
Justina Rodríguez García
}

En la historia de la industria del vidrio de Murano merece especial atención el fenómeno de expansión de esta producción suntuaria fuera de las fronteras de la República de Venecia, bien a través de la exportación o mediante la evasión de maestros al exterior. La expresión Façon de Venise, acuñada por la historiografía especializada, alude precisamente a este fenómeno de proyección europea del vidrio de Murano. Cronológicamente suele situarse a lo largo de los siglos XVI y XVII ${ }^{1}$.

Esta corriente de influencia también llegó a España, centrándose de forma particular en la corte de Madrid y su ámbito más próximo ${ }^{2}$.

La correspondencia diplomática de los embajadores españoles en Venecia con la corte de Madrid -muy en especial durante el reinado de Felipe II- documenta la entrada de piezas de vidrio de Venecia en España, junto con otra fuente de información: los inventarios de bienes de la nobleza castellana que dejan constancia de la presencia en Castilla de estas manufacturas.

Son numerosas las cartas del secretario Garcia Hernández, que sustituyó al embajador de Venecia, Francisco de Vargas, durante la estancia de éste en el Concilio de Trento, notificando, entre otros asuntos, el

\footnotetext{
1 La Façon de Venise es un aspecto de la historia del vidrio que ha interesado de forma muy especial a los investigadores, y en consecuencia es muy abundante el número de trabajos publicados sobre el tema en los distintos paises europeos en donde este fenómeno dejó su huella.

a Cataluña, y en particular Barcelona y Mataró, fueron importantes focos de manufactura de vidrio à la Façon de Venise, pero con unas características muy peculiares que pueden apreciarse en la abundante producción que ha llegado hasta nosotros. Paradójicamente, la presencia en Cataluña de maestros venecianos fue muy escasa. Para un mayor conocimiento de este tema, ver: J. Rodriguez GarciAs "La influencia del vidrio de Venecia en Cataluña», Annales du Xe Congrès de l'Association Internationale pour l'Histoire du verre. Madrid-Segovia, 1985, págs. 421-432.
} 
envío a la corte de cristalería, en su mayor parte dirigidas al propio monarca Felipe II. Destacamos algunos ejemplos:

El 3 de agosto de 1559 escribía al rey y, entre otros asuntos decía:

«(...) estos quadros con los vidrios cristalinos para hacer las vidrieras, que todo será acabado a un tiempo, y los vasos de vidrio que he comprado para bever agua y para bever vino de la manera que escrivo al secretario Gonçalo Pérez, los enviaré muy bien empacados al embaxador de Génova con persona de recaudo como vuestra Majestad me manda, para la paga de lo qual no he tomado dinero a cambio, porque lo haré de los que yo tengo de Vuestra Majestad (...) $)^{3}$.

En abril de 1562 comunicaba a Felipe II el envío de una cristalería veneciana junto con unos cuadros de Ticiano:

“(..) En primero de março embié a Génova dos caxas de vidrieras en que van 450 pieças muy buenas, no pudieron ir antes por las muchas aguas, y porque el embaxador desta Señoría no pudo llevar con su ropa los quadros que Tiziano ha hecho de Jesucristo en la oración y la Europa. Los embié ayer en una caxa muy empacados con el ordinario de Génova, encaminando todo al embaxador Figueroa, a quien embié la letra de Vuestra magestad y le screví que con la primera ocasión lo remitiese a donde Vuestra magestad le mandara y assí creo que lo hará (...)" ${ }^{4}$.

Algún tiempo después mandaba un resumen de todo el material facturado desde 1563: el 5 de octubre de 1559 fueron remitidos desde Venecia a Génova cuatro cajas de vasos de vidrio para agua y vino y dos vidrieras de cristal lustradas para ventanas, haviendo costado las vidrieras, que fueron cuatrocientas piezas, 219 escudos, y los vasos, 101, a lo que habia que añadir por gastos de embalaje y derechos de aduana, 15 escudos más. El transporte de todo esto y los cuadros de Ticiano de Cristo en el Sepulcro y Diana y Calixto suponian 45 escudos. El 15 de febrero de 1562 había comprado 450 piezas de vidrieras lustradas por 196 escudos, pagando por ellas y los cuadros del pintor veneciano Cristo en la oración y Europa, 30 escudos ${ }^{5}$.

Más tarde (21 de julio de 1563), García Hernández avisaba que se enviaban desde Génova dos cajas de vidrios para el rey y que el 27 de septiembre del mismo año habia remitido otras cinco, dos para el secretario Gonzalo Pérez y una para Gabriel de Zayas ${ }^{6}$.

\footnotetext{
3 AGS, Estado (Venecia), leg. 1325, fol. 262; 3 de agosto de 1559; Serralbo Aguareles, E. "La cultura y el arte veneciano en relación con España a través de la correspondencia diplomática de los siglos XVI-XVII", Revista de Archivos, Bibliotecas y Museos, 62, no 3, 1956, pág. 654.

4 Ibidem, leg. 1324, 10 de abril de 1562.

5 Serralbo aguareles, E., op. cit., pág. 655.

6 Ibidem, págs. 655-656.
} 
El 9 de octubre volvía a dar noticias relacionadas con la adquisición de las manufacturas venecianas ${ }^{7}$.

Los envíos continuaron durante el año 1564. El 24 de marzo, desde Venecia, el secretario García Hernández remitía a Génova tres cajas con 700 vidrieras que el arcediano Gonzalo Pérez, secretario de Felipe II, le había pedido para su Majestad ${ }^{8}$.

El 15 de julio García Hernández se dirigía al rey comunicándole que había escrito al embajador Figueroa recomendándole que pusiera un mayor cuidado en lo relacionado con la cristalería veneciana que había de mandar a España, pues en el anterior envío se había extraviado una de las cajas, que al parecer ya estaba localizada ${ }^{9}$.

Entre los gastos realizados en servicio de Su Majestad por Diego Guzmán de Silva, que ocupaba la embajada de Venecia desde 1571, estaban entre otras muchas cosas «2.500 vidrieras de tres suertes, de las de 17 , de las de 10 y de la mitad de 17 ", que eran medidas habituales de los espejeros de Murano, por las que se pagaron 850 escudos de oro, a razón de tres vidrieras por escudo ${ }^{10}$. En 1585 era embajador en Venecia D. Cristóbal de Salazar, en cuya correspondencia aparecen también noticias relacionadas con la demanda de cristalería de Murano. El 29 de enero de 1585 escribía al Duque de Terranova, gobernador de Milán, a propósito de unas copas y vasos venecianos, que éste requería, los cuales deseaba que fueran decorados con la técnica del grabado al diamante ${ }^{11}$.

Otro momento en el que queda constancia de esta relación comercial con Venecia es en el último tercio del siglo XVII, durante el reinado de Carlos II. Destacamos uno de estos encargos realizado por la reina regente doña Mariana de Austria, con motivo del acondicionamiento de las habitaciones de su hijo, el rey Carlos 11 , para lo que se dirigía al marqués de la Fuente, embajador en Venecia (2 de octubre de 1675):

\section{Ibidem, pág. 657.}

lbidem.

- Tiziano e la Corte di Spagna nei documenti dell'Archivio Generale di Simancas, Istituto Italiano di Cultura. Madrid, 1975, pág. 88.

10 AGS, Estado (Venecia), leg. 1550. Sumario de lo que se ha gastado en servicio de S. M. en Venecia desde 1571 hasta fin de 1573.

11 Dice el texto: «Muy magnifico señor: he holgado por una carta de vuestra merced de 19 de enero que aya resçevido los designos de las copas y basos, y la diligençia que pensava poner en que se hiçiesen brevemente que es lo que mucho desseo: Las copas entiendo que se han de labrar al diamante, como vuestra merced me escrive, y no tengo para qué alargarme açerca desto, pues con quedar a cargo de vuestra merced, puedo estar descuydado y esperar que se harán con toda curiosidad (...) »: AGS, Estado (Venecia), leg. 1535, fol. 19. 
«(...) Para las ventanas del quarto de mi hijo se necesita de quatro mil vidrios, los dos mil del número diez y los otros dos mil del número $17 \mathrm{y}$ assí os encargo dispongáis que en la primera ocasión que se offrezca en qué poderles conducir a España, se encaminen con toda la seguridad demandada, que quanto antes se pueden tener aquí para que sirvan a este effecto y el coste que también lo pondréis en la quenta de los semestres que embiaréis para que os pueda hacer lo bueno que importaren $(\ldots){ }^{12}$.

El embajador se responsabilizó del encargo y se comprometió a enviar el pedido lo antes posible ${ }^{13}$.

Como ya se ha indicado, a través de los inventarios de bienes se puede rastrear también la existencia en la corte de cristal procedente de Venecia. No vamos a hacer una relación exhaustiva de los numerosos ejemplos documentados. Baste señalar los más significativos como testimonio de este hecho.

De 1500 es uno de los más notables inventarios de objetos de vidrio pertenecientes a la reina Isabel la Católica. Contiene una relación de 148 piezas enviadas por el rey Fernando desde Barcelona a su esposa, que se hallaba en Alcalá de Henares. Una segunda parte del mismo inventario incluye 268 objetos, también esmaltados, que ya estaban en el camarín de la reina antes de la llegada de los de Barcelona ${ }^{14}$. La procedencia de estas piezas podría ser catalana, aunque su vinculación a Venecia se pone de manifiesto al estudiar detenidamente las descripciones.

En el primer inventario del Palacio de El Pardo, que se hizo en 1504, hay un gran lote de vidrios de Venecia, descritos con cierta minuciosidad y otro, también importante, de manufacturas de Barcelona ${ }^{15}$.

La condesa de Altamira poseía ciento veinticuatro piezas «de copas e jarros e garrafas e varrilles e taças de vidryo de Venecia», como figura en la enumeración de sus pertenencias ${ }^{16}$. Muy interesante es la tasación de los bienes muebles de $D$. Beltrán de la Cueva, tercer duque de Alburquerque, de 1560 , en la que se puede ver una notable selección de

12 AHN, Estado, libro 733; 2 de octubre de 1675.

13 Ibidem, 2 de noviembre de 1675.

14 Este documento se conserva en el Archivo General de Simancas y fue publicado por J. Gudiol RICART, Los vidrios catalanes. Barcelona, 1941.

15 SÁnchez Cantón, F. J., "El primer inventario del Palacio de El Pardo (1564)", Archivo Español de Arte y Arqueología, X, 1934, págs. 73-74.

16 «Testamento e inventario de doña Ana de Toledo, Condesa de Altamira. Año 1546», Academia Gallega, Colección de documentos históricos, 1931, vol. II, págs. 14-16. 
piezas de vidrio de Murano ${ }^{17}$. D. Juan de Borja, primer conde de Ficalla, poseía un «camarín de los vidrios y porcelanas», con vidrios de Venecia y Barcelona, junto con mesas de jaspe, corales, vidrios guarnecidos de plata y oro ${ }^{18}$. Este camarín de los vidrios era común entre los personajes de la nobleza española con aficiones coleccionistas, y se utilizaba para albergar vidrios, cristales, porcelanas, escritorios y objetos varios.

Además de la sugestiva relación de objetos del palacio de El Pardo, en la testamentaría de Felipe II hay una cuantiosa enumeración de piezas, en su mayor parte de cristal de roca y de cristal labrado con guarniciones de metales preciosos y esmaltes, algunas presumiblemente de Venecia ${ }^{19}$.

En los banquetes preparados en Sevilla en 1570 con motivo de una visita del rey y su séquito a esta ciudad, el servicio de mesa era de porcelana y cristalería de Flandes y de Venecia ${ }^{20}$.

Durante el primer tercio del XVII todavía aparecen referencias a estas manufacturas en los inventarios de la nobleza española, pero de forma menos constante. Así, en la relación de bienes de D. Agustín Álvarez de Toledo (1601) hay una larga enumeración de vidrios de Barcelona y otros tantos de Venecia ${ }^{21}$, al igual que en las de D. Gaspar Mendes de Parada, de $1608{ }^{22}$ y de Doña Francisca Ruiz de Castejón (1610), en donde los productos de Venecia alternaban con los de Barcelona, Cadalso y Florencia ${ }^{23}$.

17 «Inventario del mobiliario, alhajas, ropas, armeria y otros efectos del Excmo. Señor D. Beltrán de la Cueva, Tercer Duque de Alburquerque. Año 1560", Revista de Archivos, Bibliotecas y Museos, 9, 1883, págs. 101-102.

18 Morán, M. y Checa, F., El coleccionismo en España. Madrid, Cátedra, 1985, pág. 74.

19 Documentos inéditos que pueden servir para la Historia del arte Español. Vasos bernegales y otras diversas piezas de cristal, siglo XVI. Testamentaría de Felipe II, en El Arte Español, t. VIII, 1860, págs. 107-172.

20 MAL-LARA, J. DE, Recebimiento que hizo la muy noble y muy leal ciudad de Sevilla a la C.R.M. del rey D. Philipe N.S. Sevilla, 1570, cit. por Frothingham, A. W., Barcelona Glass in Venetian Style. Nueva York, 1956, p. 20.

21 La relación de bienes de D. Agustín Álvarez de Toledo (1601) contiene una larga lista de vidrios de Barcelona y otros tantos de Venecia (Archivo Histórico de Protocolos de Madrid, AHPM, Sebastián Aleas, prot. 2073, fol. 763, mayo de 1601).

22 En el de D. Gaspar Mendes de Parada (1608) también hay piezas de Venecia, con la correspondiente tasación:

- Dos taças de Venecia, diez y seis ducados.

- Quatro candelerillos de vidrio de Venecia, 8 ducados.

- Una copa grande de Venecia, 12 ducados.

- Otro vidrio de Venecia, dos ducados.

- Una fuente de vidrio de Venecia, dos ducados.

- Un vidrio largo de Venecia, seis ducados.

AHPM, Jerónimo Fernández, prot. 2543, fol. 564v-568r, 21 de junio de 1608.

23 Doña Francisca Ruiz de Castejón (1601) tenía vidrios de Valencia, Cadalso, Barcelona y Florencia (AHPM, J. de Urraca, prot. 1586). 
Pinheiro da Veiga, cronista portugués que estuvo en Valladolid durante el reinado de Felipe III, narra en su obra Fastiginia, con admiración y un cierto escándalo, el lujo de la corte española y la ostentación de sus fiestas. Con motivo de un convite que dio el duque de Lerma al almirante de Inglaterra, describe los aparadores para fuentes suntuosas «para vidrios de Venecia y Barcelona, barros de Portugal y botellería» ${ }^{24}$.

Flandes, en donde la Façon de Venise había tenido un notable desarrollo, fue otra fuente de la que se surtió la corte española en su demanda de cristalería al estilo de Venecia. Los propios monarcas españoles promovieron en esa parte del imperio español la manufactura de estos objetos, favoreciendo la iniciativa de numerosos maestros venecianos instalados en los principales centros vidrieros flamencos, tales como Amberes y Bruselas. Con Carlos $V$ y María de Hungría, hermana del emperador y gobernadora de los Países Bajos, así como durante el reinado de Felipe II, se concedieron numerosos privilegios a estos maestros para manufacturar vidrio à la Façon de Venise.

La presencia de un tal Cornachini, heraldo de armas del emperador Carlos V, al frente del primer establecimiento vidriero en Amberes, especializado en manufacturas inspiradas en la producción muranesa (1541), pone de manifiesto el interés del monarca español en fomentar esta actividad $^{25}$.

Transcribimos por su interés testimonial un fragmento de una carta del cardenal Granvela, gobernador de Flandes desde 1559 hasta 1564, fechada el 20 de agosto de 1563, en la que alude, precisamente, a uno de estos maestros flamencos:

"(..) Vandenesse no me ha comunicado cosa alguna de V. M., bien que no sé si la memoria que en la otra carta digo me dio que los mesmo que $V$. $M$. le podia haver encargado que me dixese, pero no me hizo mención de $V$. M. ny tampoco he visto la cédula que dize que le embió para que la persona que yo nombrasse pudiesse sacar destos estados sessenta caxas de vidrio y materias en los reynos de España en dos años, a fin que se pudiesse hazer favor al maestro de los vidrios de Envers, que cierto es hombre de bien y que ha hecho gran beneficio en estos estados de su arte, porque en lugar de los vidrios de Venecia que con gran costa y sacándose el dinero destos estados, solian traerse aquí, se hallan agora hechos aquí quasi tan buenos y

24 MoRÁn, M. y CHECA, F., op. cit., pág. 222.

25 SchuermanN, H., "Verres fabriqués aux Pays Bas à la Façon de Venise et Altare», Bulletin des Commissions Royales d'Art et d'Archéologie, 24, 1885, p. 43; PINCHART, A., "Les fabriques de verres de Venise, d'Anvers et de Bruxelles au XVI et au XVII siècle", Bulletin des Commissions Royales d'Art et d'Archéologie, 21, 1882, págs. 373-375. 
por lo menos la mitad mejor barato y ha gastado infinito para traer oficiales de Venecia y poner todas las cosas en orden antes de poder labrar lo vidrios, y pues esta cédula que se havía enbiado a Vandenesse no parece ya que Su Majestad havía hecho la merced y V.M. puesto su trabajo, recebir la he muy grande en que se me embíe un duplicado della para que este hombre de bien pueda ser ayudado y gratificado (...) ${ }^{26}$.

Este maestro de Amberes al que alude Granvela podría ser Ambrosio de Mongarda, que trabajó en dicha ciudad por esas fechas durante un largo período de 20 años, gozando del monopolio y de la protección real para manufacturar vidrios à la Façon de Venise, de cuya producción se beneficiaban, además de los propios flamencos, el monarca español y su corte. La alta calidad de la misma y su ventaja económica garantizaron su éxito, al menos en Flandes y en territorio español.

Como ya se ha indicado, otro recurso utilizado en las cortes europeas para proveerse de cristalería veneciana fue conseguir mano de obra procedente Murano, favoreciendo la creación de hornos regentados por maestros de la isla del Adriático.

En España también se ha podido documentar la presencia de estos operarios, especialmente en la corte de Madrid y sus alrededores. En el siglo XVI, sobre todo durante la segunda mitad, hay algunas noticias documentales, pero las más importantes corresponden a la siguiente centuria.

Los archivos de Venecia y los registros de Altare, importante centro vidriero en la Liguria, en donde se asentaron muchos muraneses, han aportado nombres. En el año 1544 estaban en nuestro país - sin que sepamos en qué localidad concreta trabajaron-Domenico Moro, Mattio di Piave, llamado Vissiga, y Antonio di Piave, todos ellos de Murano. Once años más tarde -1555- trabajaron en España Alvise Serena, Antonio del Sol, Marino Moro y dos miembros de la familia Ballarin, pertenecientes a ilustres familias de vidrieros muraneses ${ }^{27}$. Un tal Gaspar Veneziano estaba activo en Barcelona en $1580^{28}$.

En torno a 1600 llega a España Domingo Barovier, miembro de la más célebre dinastía muranesa de artistas del vidrio. Se instaló en Palma de Mallorca, en donde trabajó enseñando a los artesanos indígenas sus propias técnicas. Allí permaneció hasta 1607, año en el que se trasladó a la

${ }^{26}$ AGS, Casas y Sitios Reales. Consultas y providencias generales, leg. 275, fol. 27.

27 Schuermann, $H$., "Verres Façon de Venise fabriqués aux Pays-Bass", Bulletin des Commissions Royales d'Art et d'Archéologie, 29, 1890, p. 140.

28 Archivo de la Corona de Aragón (ACA), Real Patrimonio, M. R., 1460, fol. 87v, 30 de junio de 1580 . 
corte, instalándose en El Escorial con un privilegio real para trabajar ocho años al servicio del monasterio, en donde construyó el horno, junto a la enfermeria. Un año más tarde se asoció con Nicolao Palmota, originario de Ragusa, para reorganizar la pequeña manufactura, colaborando con ambos un hijo de Barovier, Juan, que firmó un contrato de oficial ${ }^{29}$.

La prematura muerte del maestro de Murano, antes de agosto de 1608 , dio al traste con el negocio, disolviéndose la sociedad. Palmota trajo a El Escorial, por cuenta propia, algunos oficiales de Cuenca con el fin de mantener el horno activo, pero estos operarios no supierori realizar los trabajos conforme a lo que Barovier había concertado con el rey Felipe III, lo que determinó la ruina definitiva de la pequeña factoría ${ }^{30}$.

A pesar de su efímera existencia, esta manufactura artesanal al servicio de la Corona reviste un especial significado como antecedente de los hornos de vidrio establecidos en España por maestros extranjeros, con ayuda de la Corona, en el último cuarto del siglo XVII, dentro del marco de recuperación económica de finales del reinado de Carlos II.

Otro miembro de la familia Serena está localizado en España en 1650. En 1688, Prospero Bertolucci, Gio Battista Pisano y Giovanni Pietro, hijo del anterior, están registrados en Altare por pagar a los cónsules de la ciudad en moneda española, lo que hace suponer que trabajaron y enseñaron su oficio en España ${ }^{31}$.

En 1685 y 1688 se instalaron aquí otros altareses: Secondino Zanettino, Varaldo y Giuseppe Perotto.

Del 11 de diciembre de 1677 es una carta del marqués de Villagarcía, embajador especial en Venecia, contestando a Jerónimo de Eguía a propósito de un maestro vidriero veneciano que querían enviar a España, en donde expresa las dificultades que conlleva dicha gestión, sobre todo de orden económico ${ }^{32}$.

En el Archivo de Estado de Venecia he podido documentar algunas noticias interesantes, datadas la mayor parte en el último tercio del XVII, relacionadas con la instalación en España de hornos à la Façon de Venise.

Hay varias cartas del embajador Rodrigo Cornaro relacionadas con la presencia en la corte de Madrid de un vidriero de Murano llamado Antonio

29 Para una mayor información sobre este tema, ver mi articulo: «Domingo Barovier, vidriero veneciano en España (1600-1608)», Espacio, Tiempo y Forma, no 4, 1989, págs. 476-500.

30 Ibidem.

31 SchuermanN, H., «Verres Façon de Venise...», op. cit., pág. 141.

32 AHN, Estado, libro 733, en G. Serralbo Aguareles, op. cit., pág. 682. 
Pellizari. El 21 de julio de 1678 el embajador notifica a las autoridades de la Serenísima que un súbdito veneciano, el aludido Pellizari, que ruyó a Francia en donde trabajó haciendo espejos, ha establecido un horno en el propio palacio real de Madrid para surtir a la corte de estas manufacturas. Cornaro, después de poner en duda la preparación profesional del individuo, alude a la presencia en la corte de otros dos maestros de nacionalidad flamenca que también elaboran cristales y vidrios, "los cuales son de gran perfección y que irán mejorando cada vez más por la facilidad y calidad óptima de los materiales que aquí en España hay, en lugar de que de estas partes se envíen a Venecia para tal trabajo» ${ }^{33}$. En agosto de 1678 volvía a dar noticias de la fábrica de espejos promovida por Pellizari, e informaba sobre el viaje que éste había realizado a Francia para traer algunos maestros expertos en esta técnica. También notificaba que se hallaban en Madrid tres maestros muraneses - Hirolamo Basfini, Antonio della Riviera y Juanne Polo- después de haberse intentado abrir camino en Francia. Transcribimos un fragmento de este documento:

«(..) Ciertamente, se han comenzado a trabajar las lámparas, que si bien son pequeñas, con el tiempo pueden llegar a tener mayor tamaño y perfección. Yo, sin embargo, estimo que ateniéndose a las prudentes reflexiones de VV. CC. en el perjuicio o en las consecuencias que puedan provenir de este interés, si no se rompe en sus comienzos de raíz con los medios que parezcan más oportunos, vigilando que aquí no llegue ninguno del arte de los specchieri a ser convencido de abandonar la patria con la ilusión de ventajas y de provechos, y por lo que respecta al susodicho Antonio Pellizari y con motivo de su liberación de los bandos que tiene o con un salvoconducto que creo sería aceptado por él, facilitarle el modo de volver a Venecia, con el fin de que al no permanecer en el extranjero, quede suprimido el fomento de los mal enfocados consejos y proyectos que la necesidad va sugiriendo para sostener el propio alimento y la vida (...)».

Y a continuación facilita información sobre otro horno en los alrededores de la corte:

«(...) Yo, entre tanto, sigo atento para facilitar a C.C. V.V. los mayores datos posibles, que me aclare cómo debo asegurar una mayor diligencia, trabajándose los vidrios y cristales en la vecindad de esta ciudad en el lugar de Machera, que son de singular exquisitez y bondad. Como, sin embargo, los operarios no son súbditos de la Señoría, sino españoles que habiendo estado por largo tiempo en Flandes, han aprendido el arte y venidos después aquí a ejercitarlo $(. . .)^{34}$.

33 Archivo di Stato di Venezia, Inquisitori di Stato, Dispacci degli Ambasciatori in Spagna, no 485. Madrid, 21 de julio de 1678.

34 Ibidem, 5 de agosto de 1678. 
El 7 de diciembre del mismo año Cornaro comunica a los Inquisitori di Stato que Pellizari se ha marchado a Lisboa después de haber puesto en evidencia su incompetencia y falta de preparación ${ }^{35}$.

En el tratado del vidriero de Valdemaqueda, Juan Danis (1676) - El Tratado de la fábrica del vidrio, de Juan Danis, y el "modo" de hacer vidrieras, de Francisco Herranz - se cita a Pellizari al hablar de las vidrieras cristalinas al estilo de Venecia:

«(...) He visto hacer esto a un veneciano Ilamado Antonio Pellizari que su Magestad imbió a este horno a que hiciesen unas vidrieras, para que el dicho Antonio las puliese delante del Rey como lo hizo. En dos de la una se hizo un espejo que está en Palacio y la otra quedó blanca, y es cierto que beneficiadas assí no le hacían betaja las de Venecia; yo lo vi hazer, que solo a esto fui a Madrid; el travajo es que aviéndose de costear en España, salen mucho más caras que dan las de Venecia, porque un hombre trabajando vien, ha menester tres días, que a seis reales son 18, y el costo de la vidriera, y se hallara de Venecia por 12 ó 14 reales, a causa de que los jornales de allá son muy cortos (...)" ${ }^{36}$.

Antonio Pellizari es paradigma de un tipo humano que debió ser bastante común en el ámbito europeo de la Façon de Venise: el aventurero que, aprovechando su origen veneciano, pretende suplantar la personalidad de uno de esos expertos maestros con el ánimo de hacer fortuna en el extranjero. Su peregrinación por Europa comenzó en Francia, continuó en España y terminó en Portugal, a donde marchó Pellizari prácticamente huido.

En la primera carta es de destacar la alusión que se hace a dos artistas de nacionalidad flamenca que estaban en Madrid y que manufacturaron "vidrio de gran perfección», debido fundamentalmente a la "óptima calidad de los materiales que aquí en España se tienen». El embajador veneciano hace una observación importante: que estos excelentes materiales deberían ser llevados a Venecia para trabajarlos allí. Ya es sabido que la barrilla de Alicante era exportada en grandes cantidades fuera de España, incluida Venecia, $y$, precisamente, la calidad y perfección de las manufacturas de la isla de Murano eran debidas en muy buena parte a los componentes de la mezcla, que se seleccionaban cuidadosamente. En estas fechas tardías del documento citado, la producción de Murano atravesaba un período de menor auge que en la centuria anterior, lo que puede justificar este comentario de Cornaro.

\footnotetext{
35 Ibidem, 7 de diciembre de 1678.

36 V. NIĖTo ALCAIDE, "El Tratado de la fábrica del vidrio, de Juan Danis y el "modo" de hacer vidrieras, de Francisco Herranz", Archivo Español de Arte, n 158, 1967, p. 298.
} 
En 1679, año en que se creó en España la Real y General Junta de Comercio, que, como es sabido, tenía como objetivo primordial el fomento de la industria, llegó a Madrid procedente de Flandes, Dieudonné Lambotte, maestro vidriero que estableció una factoría de vidrio suntuario à la Façon de Venise en San Martín de Valdeiglesias, con el apoyo real ${ }^{35}$. Trajo consigo veinticinco oficiales flamencos y venecianos, mano de obra que consideró necesaria para hacer frente a las actividades del horno y adiestramiento de los operarios españoles. Las condiciones del contrato presentadas por Lambotte fueron aprobadas por Carlos II en mayo de 1679 , ordenando a la Junta de Comercio y al Consejo poner en marcha los trámites oportunos para su cumplimiento ${ }^{37}$.

En 1680 la fábrica ya estaba en actividad y continuó su labor hasta 1683, con Lambotte al frente. En esta misma fecha el maestro flamenco - no se sabe por qué causa, aunque es probable que fuera por falta de entendimiento con la Corona- la traspasó a Antonio de Ovando, maestro vidriero de Cadalso de los Vidrios, al que concedió el monopolio de la fábrica hasta 1689 - fecha en la que terminaba el privilegio real concedido al flamenco- a cambio de obtener la tercera parte de las ganancias.

Tras la muerte de Lambotte y en el mismo año 83, la fábrica continuó su actividad con Ovando y la colaboración de otro maestro veneciano, Santiago Bartholito (castellanización de Giacomo Bertoletti), que fue el principal oficial que vino acompañando a Lambotte. Este maestro fue propuesto por D. Cristóbal de Ontañón, solicitador fiscal de las fábricas de vidrio y papel, para regentar una nueva fábrica en Galapagar. No sabemos con certeza si esta factoría llegó a ser una realidad, aunque es muy probable que coincida con la citada por el embajador veneciano en España, Gio Pesaro, en 1689, pero en un lugar llamado Torre del Marqués, que muy bien podía ser Galapagar o sus alrededores. Guillermo Toreata o Torcata, apodado "el Flamenco", era el maestro que, según el informe del embajador, estaba al frente de la manufactura, quien previamente había trabajado en un horno de vidrio veneciano en Aragón, y colaboraba con él un veneciano llamado Giacomo Bertoletti, «natural veneciano, evadido con bando capital de la Patria y desde diez años está aquí, donde se ha casado", que coincide con el nombre del maestro que vino con

37 La Junta de Comercio, que se propuso como medida económica reducir el consumo de productos extranjeros para proteger la producción propia, fomentó la venida a España de operarios europeos con el fin de renovar e impulsar la industria española. Cfr. J. Rodríguez García, "Algunas noticias sobre una fábrica de vidrio de Venecia en San Martín de Valdeiglesias (1679. 1689)”, Espacio, Tiempo y Forma, nº 4, 1989, págs. 157-158, nota 5 . 
Lambotte y que estuvo en Galapagar. Sigue informando el embajador que «en cuanto al posible éxito de tal fábrica no se deben hacer conjeturas, sino esperar a ver los resultados. Yo creo que el proponente no cumplirá con las promesas hechas a Su Majestad de producir toda la demanda que necesita esta corte, ya que se usan todas las hechuras (o modos de hacer de Venecia)" ${ }^{38}$.

En Cadalso de los Vidrios, en la provincia de Madrid, existieron hornos en donde se manufacturó vidrio veneciano de alta calidad, a juzgar por los múltiples testimonios escritos que nos han llegado. Sin embargo, siendo el centro de producción de vidrio más famoso de Castilla, está todavía por estudiar. Larruga da alguna noticia relacionada con la actividad vidriera en Cadalso:

\begin{abstract}
«En la villa de Cadalso hay dos fábricas de vidrio ordinario propias de la Excelentísima Señora Marquesa de Villena, que tienen en arrendamiento algunos vecinos de la misma villa, por quienes se labra el citado vidrio. Desde la mitad de este siglo se ha perdido un horno, y también la fama que en toda Castilla tenían sus vidrios, así por su limpieza, como por su diversidad. Si se examina el estado de esta fábrica desde el siglo pasado, se nota que en el reynado de Carlos II estaba sin uso, pues D. Antonio de Obando se encargó de restablecerla y de labrar vidrios y vidrieras como los había hecho antes (...) ${ }^{39}$.
\end{abstract}

Para terminar, conviene hacer referencia a Valdemaqueda, otro destacado centro de manufactura de vidrio, cuya tradición se remonta al menos al siglo XVI ${ }^{40}$.

Larruga dice que «en tiempos del señor Felipe IV eran muy apreciados los vidrios de Valdemaqueda, pero en el reinado de Carlos II todo se perdió" ${ }^{41}$. Esta noticia es inexacta, pues sabemos que precisamente en 1680 , reinando Carlos 11 , los vidrios de Valdemaqueda se vendían en la provincia de Madrid. Este dato está presente en una tarifa de precios «de alquileres, mercaderías, salarios y jornales de Madrid». En la tasación general se enumeran los "Vidrios de Venecia y otras partes", los cuales están detallados así:

«Cada vidrio de hechurillas, a dos reales y medio.

Cada vidrio de a vez, a quatro reales.

Cada copa labrada y otras hechuras particulares, a seis reales.

Cada vidrio de hechurillas de Barcelona y contrahecho de Venecia, de Valdemaqueda y Villafranca, a catorce quartos.

Cada vidrio de a vez de Barcelona y Valdemaqueda y Villafranca, siendo contrahechos de Venecia, veinte quartos.

\footnotetext{
J. Rodríguez Garcia, «Algunas noticias...», op. cit., págs. 167 y ss. 13.215, año 1679.

larruga Boneta, E., op. cit., t. X, p. 53.

L. Pérez Bueno, Vidrios y vidrieras. Barcelona, 1942, pág. 97.

LarRuga y Boneta, E., op. cit., t. XX, p. 195.
} 
Copas labradas y otras hechuras particulares de Barcelona, Valdemaqueda y Villafranca, contrahechos de Venecia, quatro reales». ${ }^{42}$.

La expresión «contrahecho de Venecia» que aparece en esta tarifa de precios alude a las manufacturas à la Façon de Venise, lo que es indicativo de un nivel selecto en la producción de estos hornos. Es probable que alguno de estos objetos procediera del horno que en 1674 habían establecido en Valdemaqueda los maestros Juan Danis y Francisco Herranz, cuyo objetivo primordial era manufacturar las vidrieras que quedaban por hacer en la catedral de Segovia, tarea que mantuvo activos a ambos hasta $1689{ }^{43}$. Estos dos maestros han pasado a la posteridad, fundamentalmente, por el tratado sobre la fabricación de vidrio y la técnica para elaborar las vidrieras, que ambos redactaron en 1676 por encargo del Cabildo, al que anteriormente se ha aludido. Cada uno redactó un tratado: Danis centró su estudio en la tecnología del vidrio (tanto para objetos huecos como vidrio plano): materiales, instrumentos, proceso de fabricación y obtención de colores. Herranz se encargó de lo relativo a las vidrieras: modo de pintarlas, cortarlas y demás facetas de su elaboración.

Los dos tratados forman parte de una misma obra y se complementan entre sí. El texto va acompañado de unos dibujos muy expresivos e ingenuos que representan los hornos y los instrumentos del vidriero, obra de Herranz, como el mismo Danis dice en el texto ${ }^{44}$. La finalidad de estos escritos era fundamentalmente recoger de forma sencilla y clara las experiencias y conocimientos técnicos de estos dos maestros con el fin de que no se perdieran.

Danis dice al hablar del «vidrio christal» (una de las fórmulas venecianas que mayor fama alcanzaron): "y éste es el christal que hazen en Francia, Flandes y otras partes y oy en San Martín, y el que se ha labrado en Vista Vella de Aragón». Esta alusión al horno aragonés se refiere muy probablemente al que Toreata estableció en Aragón, y que cerró después por falta de oficiales expertos. También es interesante la alusión a San Martín de Valdeiglesias dos años antes de que Dieudonné Lambotte abriera su factoría en dicha localidad, lo que evidencia la existencia de hornos al estilo de Venecia en este lugar antes de 1679, muy posiblemente promovidos por la Corona y dirigidos por mano de obra veneciana o flamenca.

42 Tasa general de alquileres, mercaderías, salarios y jornales de Madrid. Año 1688. Biblioteca Nacional. Varios especiales, 195.

43 V. NiETo AlCAIDE, op. cit., págs. 274 y 278 . Una copia del manuscrito original se conserva en el Museo de la catedral de Segovia.

44 loidem, pág. 278. 